

open research
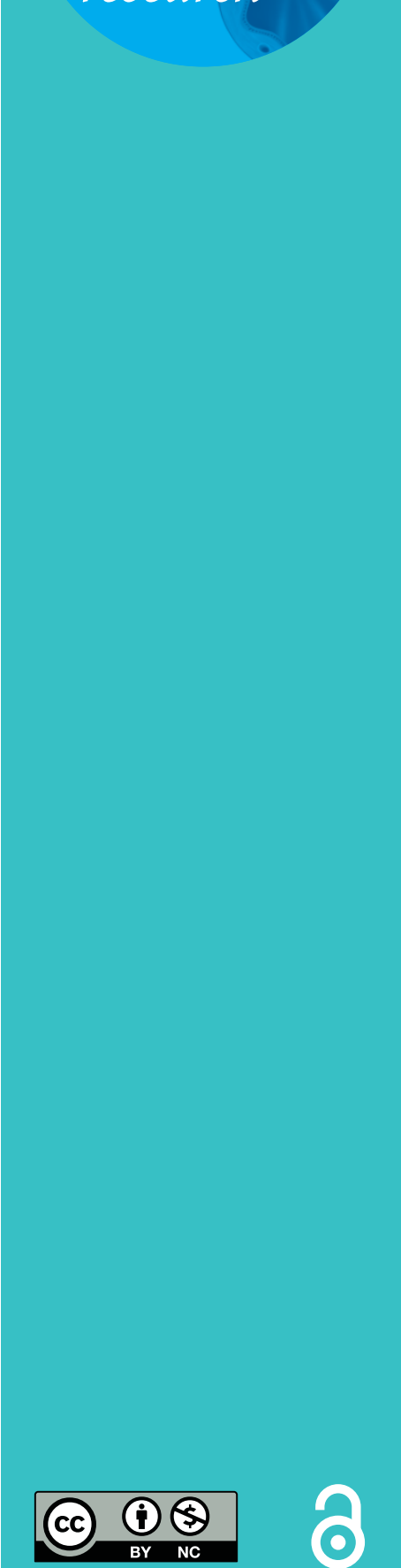

\section{Advance care planning in life-threatening pulmonary disease: a focus group study}

\author{
Nina Elisabeth Hjorth ${ }^{1,2}$, Dagny Faksvåg Haugen ${ }^{3,4}$ and \\ Margrethe Aase Schaufel ${ }^{5,6}$
}

Affiliations: 'Sunniva Centre for Palliative Care, Haraldsplass Deaconess Hospital, Bergen, Norway. ${ }^{2}$ Palliative Care Team, Centre for Pain Management and Palliative Care, Haukeland University Hospital, Bergen, Norway. ${ }^{3}$ Regional Centre of Excellence for Palliative Care, Western Norway, Haukeland University Hospital, Bergen, Norway. ${ }^{4}$ Dept of Clinical Medicine K1, University of Bergen, Bergen, Norway. ${ }^{5}$ Dept of Thoracic Medicine, Haukeland University Hospital, Bergen, Norway. ${ }^{6}$ Research Unit for General Practice, Uni Research Health, Bergen, Norway.

Correspondence: Nina Elisabeth Hjorth, Sunniva Centre for Palliative Care, Haraldsplass Deaconess Hospital, Postbox 6165, 5892 Bergen, Norway. E-mail: nina.elisabeth.hjorthaharaldsplass.no

ABSTRACT Advance care planning (ACP) is a communication process for mapping a patient's wishes and priorities for end-of-life care. In preparation for the introduction of ACP in Norway, we wanted to explore the views of Norwegian pulmonary patients on ACP.

We conducted four focus group interviews in a Norwegian teaching hospital, with a sample of 13 patients suffering from chronic obstructive pulmonary disease, lung cancer or lung fibrosis. Analysis was by systematic text condensation.

Participants' primary need facing end-of-life communication was "the comforting safety", implying support, information and transparency, with four underlying themes: 1) provide good team players; 2) offer conversations with basic information; 3) seize the turning point; and 4) balance transparency. Good team players were skilled communicators knowledgeable about treatment and the last phase of life. Patients preferred dialogues at the time of diagnosis and at different "turning points" in the disease trajectory and being asked carefully about their needs for communication and planning. Transparency was important, but difficult to balance.

ACP for patients with life-threatening pulmonary disease should rest upon an established patient-doctor/nurse relationship and awareness of turning points in the patient's disease progression. Individually requested and tailored information can support and empower patients and their relatives.

@ERSpublications

Advance care planning may increase patients' feeling of "a comforting safety", meeting their need for support, information and transparency http://ow.ly/DMQJ30jdIPt

Cite this article as: Hjorth NE, Haugen DF, Schaufel MA. Advance care planning in life-threatening pulmonary disease: a focus group study. ERJ Open Res 2018; 4: 00101-2017 [https://doi.org/10.1183/ 23120541.00101-2017].

Received: Aug 152017 | Accepted after revision: March 242018

Copyright $\odot$ ERS 2018. This article is open access and distributed under the terms of the Creative Commons Attribution Non-Commercial Licence 4.0. 


\section{Introduction}

Advance care planning (ACP) is a structured communication process enabling individuals to define goals and preferences for future medical treatment and care, to discuss these goals and preferences with family and healthcare providers and to record and review these preferences if appropriate [1]. During one or more conversations, physical, social, psychological and spiritual aspects of life are discussed [2]. Studies have shown that using a list of questions as a guide is better than using a paper-based scheme and tick boxes [2]. Important details can be outlined in a document, often as part of the medical record, and may be reconsidered and altered if the patient changes his/her mind [2].

ACP is in common use in several English-speaking countries, and effects on improved care and quality of life for both patients and their relatives have been demonstrated [2, 3]. So far, ACP has not been introduced in any Norwegian hospital, but Norwegian health authorities have encouraged research on $\mathrm{ACP}$ and pointed to the need for implementation of guidelines and advisory material $[4,5]$. In the general population, the concept of ACP is mostly unknown.

Patients with advanced incurable lung diseases are a diverse group, comprising patients who may have been living with chronic obstructive pulmonary disease (COPD) for years [6,7], patients with an insidious progression of pulmonary fibrosis [8] and patients with lung cancer who may have galloping trajectories with only months between the time of diagnosis and death [9]. All these patients often have burdensome symptoms such as dyspnoea and pain, and they often share a feeling of their life being threatened, hence, planning for the best possible care at the end of life (EoL) is important [10-12]. Believing ACP to be a feasible tool for Norwegian healthcare professionals and their patients, and working particularly with patients with advanced lung diseases (authors NEH and MAS), we designed a study to explore pulmonary patients' needs and preferences regarding ACP in order to prepare for the introduction of ACP in Norwegian hospitals.

\section{Material and methods}

We conducted a focus group study interviewing patients with advanced pulmonary disease $[13,14]$.

\section{Participants and study setting}

The focus group interviews took place at the outpatient clinic of the Department of Thoracic Medicine at Haukeland University Hospital in Bergen, Norway. This department has 17000 outpatient consultations and 3000 in-patient admissions per year. All participants were recruited from this department.

The recruitment period lasted from January 2014 to February 2015. Patients receiving treatment for advanced lung cancer, COPD or lung fibrosis were invited to participate by a dedicated research nurse, other nurses on the ward or at the outpatient clinic, or attending physicians. 42 patients were asked to participate. 17 (40\%) agreed, but only 13 participated, due to the worsening condition of the others. As soon as a group was established through successive recruitment, an interview took place. We strived for a purposive sample, aiming for diversity in age, sex, diagnosis and education. Our final sample consisted of six males and seven females, distributed between four focus groups. 12 patients were Norwegian and one was Danish. Inclusion and exclusion criteria are listed in table 1.

\section{Ethical statement}

The study was approved by the regional committee for medical and health research ethics of Western Norway (REK number 2013/1479). Written informed consent was obtained from all participants. To

\section{TABLE 1 Inclusion and exclusion criteria for focus group participants}

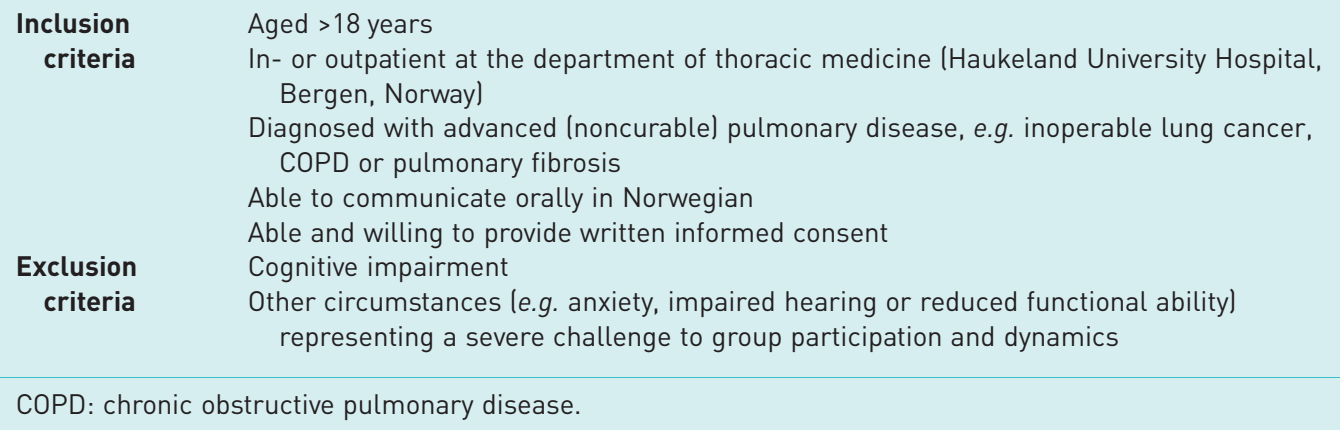


secure confidentiality, pseudonym participant names were used in the transcription and analysis, and patients were asked specifically not to share information about other co-informants elsewhere. Audio files from the interviews were stored safely on a research server belonging to the University of Bergen.

\section{Data collection}

NEH served as facilitator in all interviews and MAS acted as secretary taking field notes. No relatives attended the groups. The facilitator invited participants to talk about EoL issues and communication preferences regarding these. To balance eager participants against the more modest, we guided the discussions using questions, both to the whole group and directly to some of the participants. The interviews lasted 49-66 min and were transcribed verbatim by a secretary. Data collection was closed after four focus group interviews, as we assessed the data as sufficient to illuminate the research topic according to the concept of information power [15].

In addition to the data collected in the focus group interviews, demographic and medical information was taken from the medical record and registered in a non-identifiable form to characterise the study population.

\section{Analysis}

Data analysis was performed in collaboration between the authors NEH and MAS, using systematic text condensation, a cross-case thematic analysis suitable for both focus group data and individual semi-structured interviews [16]. The analysis proceeded through the following stages: 1) reading all the material to obtain an overall impression, bracketing previous preconceptions; 2) identifying units of meaning, representing different aspects of the patients' views on and experiences of ACP; 3) condensing and abstracting the meaning within each of the coded groups; and 4) summarising the contents of each code group to generalised descriptions and concepts reflecting the most important needs and perspectives regarding ACP as reported by the informants. Analysis was done stepwise with new interviews supplementing the sample, and a decision trail documented the choices during the analytical process [17].

\section{Results}

Having an advanced life-threatening pulmonary disease gave the patients a need for security concerning treatment and human relationships, in hospital as well as well as in private life. The participants' primary need facing EoL communication was "the comforting safety", implying support, information and transparency, with four underlying themes: 1) provide good team players; 2) offer conversations with basic information; 3) seize the turning point; and 4) balance transparency. Good team players were skilled communicators knowledgeable about treatment and the last phase of life. Patients preferred dialogues at the time of diagnosis and at different "turning points" in the disease trajectory, and being asked carefully about their needs for communication and planning. Transparency was important, but difficult to balance.

Demographic and disease-related information for the focus group participants is presented in table 2 and figure 1. All citations from participants are presented with pseudonyms.

\section{Provide good team players}

"The comforting safety" could be established by providing the patient with good team players. The participants talked about the importance of having a network of people who were knowledgeable, supportive and caring. These team players were found both in their own family, among friends and among healthcare professionals. At the hospital, the participants wanted to be offered ACP conversations, as expressed by a woman in her fifties:

I wish there was an option when you received the message that you have limited time left to live; that a doctor could talk with me about the situation and what worries me. (...) It is difficult to talk about this, but I think it can help me to talk with someone knowledgeable. (Suzanna, lung cancer)

Additionally, knowing they had good relationships with professionals in the hospital and a reliable way to get in contact with them, now and in the future, was important. Everybody in the focus groups would prefer the Department of Thoracic Medicine to offer conversations about the last phase of life. However, they pointed to the need for delicacy and respect when extending an invitation to such conversations, giving the invited patient the possibility of accepting or rejecting participation. They would prefer to have EoL conversations with people they already knew in the department, regardless of profession; it could be a nurse, a doctor or a psychologist. According to the participants, a professional having knowledge about both the patient and his/her disease was considered to be the right person in the healthcare system to talk to, including when it came to conversations about the last phase of life. Some argued the age difference should not be too big between patient and healthcare professional; indicating that they preferred a person either about their own age or older. The quality of the relationship and rapport were the most important 


\section{TABLE 2 Demographic and disease-related information for focus group participants}

Age years

$65(52-80)$

Sex

Female

7

Male

Occupation

Shop assistant/office worker/factory worker

Academic

Seaman

Self-employed

Living situation

Alone

With spouse/partner

Disease

Small cell lung cancer disseminated disease

Nonsmall cell lung cancer stage III-IV

COPD GOLD criteria stage IV

Idiopathic pulmonary fibrosis

Treatment

Inhalation therapy

Chemotherapy

Immune-modulating therapy

Radiation therapy

\section{Comorbidities}

Asthma, COPD, emphysema, bronchiectases, OSAS

Chronic pain, osteoporosis and/or arthrosis

Coronary heart disease

Diabetes mellitus

Dermatological diseases

Other malignancies

Renal failure

\section{Medication}

Nonopioid analgesics

Opioid analgesics

Corticosteroids

Benzodiazepines

Antiemetics

Laxatives

Antidiabetics

Inhalation aerosol

Antihypertensives, statins, diuretics or nitrates

WHO performance status

Data are presented as mean (range) or n. COPD: chronic obstructive pulmonary disease; GOLD: Global Initiative for Chronic Obstructive Lung Disease; OSAS: obstructive sleep apnoea syndrome; WHO: World Health Organization.

aspects when choosing a team player; the sex of the individual followed. The most important qualification when offering EoL conversations was being competent in supportive teamwork and clinical communication, making the patient feel safe and understood while at the same time imparting important information:

I don't think it is painful to talk about dying as long as I have a person teaming up with me, and who really knows what it is all about. (Nancy, lung cancer)

Most participants emphasised that it might be painful and difficult to talk about EoL issues. Several mentioned they were not capable of talking about everything on their mind with their nearest and dearest, or abstained from doing so in order not to hurt them. Several stories referred in particular to situations at times of disease worsening, when patients wanted a conversation with relatives, facilitated by healthcare professionals: 




FIGURE 1 Symptom scores for the study participants using the Edmonton Symptom Assessment System, revised (ESAS-r), a numerical rating scale. 0: no symptom; 10: worst possible symptom. Data are presented as mean (range).

I am rather open minded, we are open minded at home, but I don't manage to talk about death. I think it may be easier to talk about death with someone who's on the outside. (Gary, COPD)

Offer conversations with basic information

Many participants stressed the importance of receiving the information they needed. Information about present and future symptom management was important for most of the participants. Several mentioned anxiety-provoking dyspnoea as well as fearing future pain and other distressing symptoms. They wanted information about topics such as practical aids, medications, legal rights and social benefits, finance and who to contact when they needed help. Talking about challenges and services available for the last phase of life was mentioned as a prerequisite for good quality of life:

Because I have limited time left to live, I want to know everything that may happen to me. I want healthcare professionals in the hospital to inform me of my rights and benefits, the expected disease trajectory and the last phase of life in order to fulfil my wish for dignity at the end of life. (Nancy, lung cancer)

Others expressed a wish for thorough information in the future, underlining that they did not feel ready for it yet. Some emphasised how important it was that doctors did not give wrong information, but ensured adequate knowledge about treatment and side-effects, enabling patients to make decisions in line with their own preferences. The participants did not talk much about future planning. Yet, when thinking about a possible future situation when they no longer could make decisions for themselves, some of them mentioned a wish for a proxy:

My children shall be my attorneys if I should become so ill I am no longer in control over my own thoughts; so that I will not receive more treatment than I would have wanted. (Paul, lung cancer)

Participants described how information about the disease as well as social benefits supported relatives in a difficult situation. Some expressed difficulties knowing what to ask for when they had an appointment with the doctor, but at the same time wished to know what was happening to them. In addition, they described the immense challenge of being severely ill, including reduced capacity to remember all eventualities and needs. Thus, they wished for a proactive approach from the healthcare system, with the option to refuse an offer of conversation rather than having to ask for it themselves when they already were so heavily burdened, outlined like this:

I have so many thoughts on my mind now that I'm ill, so it is difficult to remember what I should ask about; it is better that they offer information about what they think I need to know. (Evangeline, lung cancer)

\section{Seize the turning point}

Most participants wanted a tailored EoL dialogue at the time of diagnosis. However, the COPD patients suggested that their entering the most serious disease stage (stage IV) would be a suitable time for an ACP conversation, even if they might have several years left to live. All participants underlined that an individual approach was mandatory, respecting each patient's particular needs: 
I think there is a need for more than one conversation, and that we could be offered one in the beginning, then one or two later on when the condition is getting worse. It must be an individual approach. (Peter, pulmonary fibrosis)

It was also important that they received a "warning shot" in advance, to be able to prepare for the conversation mentally and practically, and especially to invite persons they wanted to be present. They regarded it as important that ACP conversations were held while the patient was cognitively sound, and that distressing symptoms such as dyspnoea and pain were well treated beforehand. Some participants wished for an early informative and prognostic dialogue, before they became so ill that it was difficult to make a prospective care plan. One patient described how she had been struggling alone without help from healthcare professionals after having received her diagnosis, searching to find resilience and unable to talk with her close ones. After some years, she had finally found a sort of balance:

I wanted a conversation about diagnosis and prognosis much earlier in the trajectory than what I have been offered. Now, after four years, it is too late. (Evangeline, lung cancer)

Even when patients themselves received adequate and successive disease-related information, this was not necessarily enough to establish a common ground and understanding of EoL issues in their families. Several participants expressed the need for more help informing their closest ones about their diagnosis and prognosis. The participants named key turning points that often were consistent with major medical changes. Examples of such turning points were an infection triggering change, a new metastasis, increasing pain, increasing dyspnoea, loss of a function, decline in their general condition and stopping chemotherapy. At the time of treatment changes, several had wished for thorough information in order to reduce anxiety:

It is something about change; that every change can increase anxiety. (Nathan, COPD)

\section{Balance transparency}

Some participants wanted open and honest conversations about their disease, yet acknowledging it could be difficult to find a proper balance. It was upsetting if friends, neighbours and other acquaintances avoided meeting them because they felt it was difficult to talk with them. Transparency through honest conversations about their state of health and feelings was regarded very important:

Being open about everything concerning me and my family makes me feel secure. I feel safe and I feel that I make those around me safe, too, by being open. (Nancy, lung cancer)

Some needed transparency about their prognosis to be able to spend their remaining time with their family in the best way. Receiving news about limited time left to live could yield possibilities for preparing themselves as well as family members practically and psychologically for the inevitable course of the disease. Many wanted full openness and information about their disease in order to correctly inform children and grandchildren, even though this could be difficult to do by themselves:

In the nights, I cried in bed alone without telling everything to my family, but now I no longer try to hide it. (Evangeline, lung cancer)

There was broad agreement that healthcare professionals should ask the individual patients in a careful and respecting manner about their need for information and for ACP, as this could be very different from one patient to another. Documenting all given information in the patient's medical record, including any restrictions on what the patient wished to know, was regarded mandatory to avoid information errors:

The information I've received must be documented in my medical record, as well as what I don't want to know about my disease. I would find it horrible if I was given more information than I wanted. So I think medical records should clearly state the patient's preferred details and content of information. (Miranda, lung cancer)

\section{Discussion}

Facing incurable pulmonary disease, our informants described the need for a safe foundation and comforting understanding provided by EoL discussions with knowledgeable healthcare professionals at important turning points during the disease trajectory. Here, we discuss the impact of our findings and the strengths and limitations of this study.

\section{Discussion of the results}

Our results are in line with previous research demonstrating that most patients offered ACP conversations want them $[9,18]$. The challenges in finding the desired balance in transparency, both for healthcare 
professionals and patients, has been mentioned by Siouta et al. [19]. The concept of the "comforting safety" created by tailored ACP conversations and how it may improve quality of life among Norwegian patients with advanced pulmonary disease have not been demonstrated before.

This study expands our knowledge about ACP for pulmonary patients by showing that good relationships between patients and their healthcare providers are important for the basic trust needed to address sensitive themes in an ACP conversation. Patients need a tailored approach, both when it comes to the invitation to ACP conversations as well as the choice of themes. As the participants pointed out, turning points of the disease trajectory: at the time of diagnosis, a serious infection, progression of the disease, loss of a function or change in therapy, can bring uncertainty and anxiety. Awareness of these turning points is important in order to comprehend patients' special needs and vulnerability, and to seize the opportunity to introduce ACP conversations. This finding coincides with the recommendations in the Norwegian Action Programme for Palliative Care [5].

Cultural differences are important factors to consider when starting an ACP programme [20]. Since ACP has never been used in a Norwegian hospital before, the Norwegian context needed to be explored. This study demonstrates important attitudes and viewpoints on ACP conversations in Norway before any ACP programme has been started.

Whether or when it is appropriate to communicate about the last phase of life is not always obvious. LOVELl and Yates [21] found that factors influencing ACP are complex and multifaceted. Both patients' and doctors' attitudes towards the diagnosis and their understanding of the prognosis determine whether to start an ACP conversation or not. SiOUTA et al. [19] found that patients with chronic heart failure and COPD are quite unlikely to participate in discussions concerning EoL issues, partly because it is more complicated to initiate such conversations for patients with a less certain prognosis [22-24]. VERMYLEN et al. [25] found that doctors avoid conversations about ACP with patients suffering from COPD due to unique communication barriers, e.g. lack of prognostic factors and difficulty of predicting which patients are at the highest risk of premature death. Although patients may not initially be interested in discussing advance directives with their doctors, many patients still have unexpressed wishes that may not be respected if the conversation is not broached delicately. In addition, the public understanding of these diseases is not directly linked to dying, so including EoL issues in conversations can elicit negative reactions from patients $[26,27]$. Our patients pointed to the need to give the invitation to ACP conversations in a gentle manner. They expressed the need for a tailored approach, both concerning the invitation to discuss ACP, and the choice of themes.

As JABBARIAn et al. [28] have pointed out in a recent review, ACP is surprisingly uncommon in chronic respiratory disease. SIOUTA et al. [19] have remarked on the implications of not having ACP conversations: the scarcity of patient-doctor discussions concerning treatment options and preferences, and the frequent total absence of discussions on EoL issues result in less informed patients. Many patients search for relevant information on the web, which may make them challenge or question medical decision making [29]. With the increasing claim for patient autonomy and shared decision-making, ACP can be a tool to facilitate conversations that may cover this claim.

Some recent trials of clinical EoL communication have taken place in Norway, with promising results [18]. Even so, there is a need for better mapping of similarities and differences among the various groups of patients with advanced disease in Norway, and we will still have to look to experiences from other countries where ACP is incorporated into the general healthcare system [30]. Our informants wanted to be offered ACP conversations, but found it difficult to know what to ask for. Thus, the question is more about form and content than about having such conversations or not.

\section{TABLE 3 What is known about advance care planning (ACP)? What does this study add?}

What is known? ACP is used in many countries, and most patients offered conversations want to discuss it

Using a list of questions as a guide is better than using a paper-based scheme and tick-boxes only

Patients' and relatives' satisfaction with end-of-life care increases with the use of ACP

Transparency is difficult to balance

What does

this study

ACP for Norwegian patients with life-threatening pulmonary disease should rest upon an established patient-doctor/nurse relationship

add?

ACP is perceived to increase the patient's feeling of "a comforting safety", meeting their need for support, information and transparency

ACP conversations involving both patient and relatives may support the relatives in their role as good team players Turning points of the disease trajectory are times when patients may be especially in need of ACP conversations 


\section{Strengths and limitations}

A strength of our study is that the interviewer had extensive experience in EoL conversations, both as a pulmonologist and palliative care specialist. This experience guided the follow-up questions and influenced the analytical process. Additionally, we believe that the interviewer's clinical experience created an openness towards contrasts and nuances in the participants' stories. Some of the participants had been in contact with the hospital's palliative care team, but they had not met the interviewer as a palliative care physician.

Exposing vulnerability in a group, compared to individual interviews, may limit data collection, but might at the same time expand the process, as one participant's reflections may contribute to an open atmosphere giving co-participants the courage to talk. The latter was observed in all groups. Hence, we believe that, facilitated by group reflection, the participants' experiences were presented without excessive concern about making a favourable impression.

The process of recruitment was challenging and had a span of $\sim 1$ year. Many patients refused to participate, for various reasons. In addition, $23 \%$ of possible participants did not manage to come to the focus groups, mainly because of a worsening of their conditions. Many other patients were never asked to join the study, as both doctors and nurses found it difficult to raise the topic of the study. Consequently, we might have been able to recruit patients more open to, and more interested in, talking about sensitive themes, which is a possible limitation of our study. Even so, the sample recruited was representative of the clinic, and the participants represented variations in age, sex and diagnosis and family, working and living conditions; adding external validity to the findings to other hospitals treating patients with incurable lung disease in the same cultural context. Table 3 summarises current knowledge about ACP.

\section{Conclusion}

As far as possible, ACP for patients with life-threatening pulmonary disease should rest upon an established patient-doctor/nurse relationship and awareness of turning points in the patient's disease progression. Healthcare professionals can support and empower patients and their relatives by providing individually requested and tailored information. ACP may strengthen patients' resilience during the disease trajectory.

Acknowledgements: We thank the brave and honest patients for giving important information about their needs. In addition, we thank our cooperating colleagues at the Department of Thoracic Medicine (Haukeland University Hospital, Bergen, Norway) for their help and support: the nurses Margunn Gravdal, Ingunn Samnøy, May-Elisabeth Gilja, Gerd Eli Dale and Bodil D. Timberlid, and doctors Birger N. Lærum, Andreas Thelle, Trygve Jonassen and Øystein Fløtten. We acknowledge the advice given by Katrin Sigurdardottir (Haraldsplass Deaconess Hospital, Bergen, Norway) and the professional assistance from the secretaries at the Regional Centre of Excellence for Palliative Care, Western Norway.

Support statement: The study received financial support from Western Norway Regional Health Authority. N.E. Hjorth has received a PhD grant from the Sunniva Foundation, Bergen Deaconess Foundation Group. Funding information for this article has been deposited with the Crossref Funder Registry.

Conflict of interest: None declared.

\section{References}

1 Rietjens JAC, Sudore RL, Connolly M, et al. Definition and recommendations for advance care planning: an international consensus supported by the European Association for Palliative Care. Lancet Oncol 2017; 18 : e543-e551.

2 Thomas K, Lobo B. Advance Care Planning in End of Life Care. 1st Edn. Oxford, Oxford University Press, 2011.

3 Heyland DK, Allan DE, Rocker G, et al. Discussing prognosis with patients and their families near the end of life: impact on satisfaction with end-of-life care. Open Med 2009; 3: e101-e110.

4 Helsedirektoratet. Beslutningsprosesser for Begrensning av Livsforlengende Behandling. [Decision making Processes for Limitation of Life-Sustaining Treatment]. 2013. https://helsedirektoratet.no/Lists/Publikasjoner/ Attachments/67/IS-2091-Beslutningsprosesser-ved-begrensning-av-livsforlengende-behandling.pdf

5 Helsedirektoratet. Nasjonalt Handlingsprogram for Palliasjon i Kreftomsorgen. [Norwegian Action Programme for Palliative Care]. Oslo, Norwegian Directorate of Health, 2015.

6 WebMD. What are the stages of COPD? www.webmd.com/lung/copd/gold-criteria-for-copd\#1 Date last accessed: March 23, 2018. Date last updated: December 12, 2016.

7 Janssen DJ, Engelberg RA, Wouters EF, et al. Advance care planning for patients with COPD: past, present and future. Patient Educ Couns 2012; 86: 19-24.

8 Lewis D, Scullion J. Palliative and end-of-life care for patients with idiopathic pulmonary fibrosis: challenges and dilemmas. Int I Palliat Nurs 2012; 18: 331-337.

9 Ford DW, Koch KA, Ray DE, et al. Palliative and end-of-life care in lung cancer: diagnosis and management of lung cancer, 3rd ed: American College of Chest Physicians evidence-based clinical practice guidelines. Chest 2013; 143: e498S-e512S.

10 Gysels MH, Higginson IJ. The lived experience of breathlessness and its implications for care: a qualitative comparison in cancer, COPD, heart failure and MND. BMC Palliat Care 2011; 10: 15. 
11 Landers A, Wiseman R, Pitama S, et al. Patient perceptions of severe COPD and transitions towards death: a qualitative study identifying milestones and developing key opportunities. NPJ Prim Care Respir Med 2015; 25: 15043.

12 Stenzel NM, Vaske I, Kühl K, et al. Prediction of end-of-life fears in COPD - hoping for the best but preparing for the worst. Psychol Health 2015; 30: 1017-1034.

13 Malterud K. Fokusgrupper som Forskningsmetode for Medisin og Helsefag. [Focus Groups as Research Method for Medical and Health Care Studies]. Oslo, Universitetsforlaget, 2012.

14 Malterud K. Kvalitative Metoder i Medisinsk Forskning. En Innføring. [Qualitative Methods in Medical Research. An Introduction]. Oslo, Universitetsforlaget, 2011.

15 Malterud K, Siersma VD, Guassora AD. Sample size in qualitative interview studies: guided by information power. Qual Health Res 2015; pii: 1049732315617444.

16 Malterud K. Systematic text condensation: a strategy for qualitative analysis. Scand J Public Health 2012; 40: 795-805.

17 Whitehead L. Enhancing the quality of hermeneutic research: decision trail. J Adv Nurs 2004; 45: 512-518.

18 Friis P, Førde R. Advance care planning discussions with geriatric patients. Tidsskr Nor Laegeforen 2015; 135: 233-235.

19 Siouta N, van Beek K, Preston N, et al. Towards integration of palliative care in patients with chronic heart failure and chronic obstructive pulmonary disease: a systematic literature review of European guidelines and pathways. BMC Palliat Care 2016; 15: 18.

20 Rietjens JA, Korfage IJ, Dunleavy L, et al. Advance care planning - a multi-centre cluster randomised clinical trial: the research protocol of the ACTION study. BMC Cancer 2016; 16: 264.

21 Lovell A, Yates P. Advance care planning in palliative care: a systematic literature review of the contextual factors influencing its uptake 2008-2012. Palliat Med 2014; 28: 1026-1035.

22 Duenk RG, Heijdra Y, Verhagen SC, et al. PROLONG: a cluster controlled trial to examine identification of patients with COPD with poor prognosis and implementation of proactive palliative care. BMC Pulm Med 2014; 14: 54 .

23 Connors AF Jr, Dawson NV, Thomas C, et al. Outcomes following acute exacerbation of severe chronic obstructive lung disease. The SUPPORT investigators (Study to Understand Prognoses and Preferences for Outcomes and Risks of Treatments). Am J Respir Crit Care Med 1996; 154: 959-967.

24 Fruchter O, Yigla M. Cardiac troponin-I predicts long-term mortality in chronic obstructive pulmonary disease. COPD 2009; 6: 155-161.

25 Vermylen JH, Szmuilowicz E, Kalhan R. Palliative care in COPD: an unmet area for quality improvement. Int $J$ Chron Obstruct Pulmon Dis 2015; 10: 1543-1551.

26 Coventry PA, Grande GE, Richards DA, et al. Prediction of appropriate timing of palliative care for older adults with non-malignant life-threatening disease: a systematic review. Age Ageing 2005; 34: 218-227.

27 Small N, Gardiner C, Barnes S, et al. Using a prediction of death in the next 12 months as a prompt for referral to palliative care acts to the detriment of patients with heart failure and chronic obstructive pulmonary disease. Palliat Med 2010; 24: 740-741.

28 Jabbarian LJ, Zwakman M, van der Heide A, et al. Advance care planning for patients with chronic respiratory diseases: a systematic review of preferences and practices. Thorax 2018; 73: 222-230.

29 Wald HS, Dube CE, Anthony DC. Untangling the Web - the impact of internet use on health care and the physician-patient relationship. Patient Educ Couns 2007; 68: 218-224.

30 Advance Care Planning Australia. A Routine Part of Health and Personal Care. http://advancecareplanning.org.au/ Date last accessed: March 23, 2018. Date last updated: 2018. 